\title{
ON MINIMAL LOG DISCREPANCIES AND KOLLÁR COMPONENTS
}

\author{
JOAQUÍN MORAGA
}

\begin{abstract}
In this article we prove a local implication of boundedness of Fano varieties. More precisely, we prove that $d$-dimensional $a$-log canonical singularities, with standard coefficients, which admit an $\epsilon$-plt blow-up have minimal log discrepancies belonging to a finite set which only depends on $d, a$ and $\epsilon$. This result gives a natural geometric stratification of the possible mld's in a fixed dimension by finite sets. As an application, we prove the ascending chain condition for minimal log discrepancies of exceptional singularities. We also introduce an invariant for klt singularities related to the total discrepancy of Kollár components.
\end{abstract}

\section{Contents}

1. Introduction 1

Acknowledgements 3

2. Preliminaries 3

2.1. Singularities 3

2.2. Bounded families 5

2.3. Finite morphisms $r$

2.4. Complements 10

2.5. Examples 11

3. Proof of the main Theorem 12

References 13

\section{INTRODUCTION}

The development of projective birational geometry has been deeply connected with the understanding of singularities [Xu17]. In particular, global theories shed light on the singularities of the minimal model program [Kol13]. However, singularities of dimension greater than three seem too complicated to have an explicit characterization [Kol16]. Therefore, a more qualitative and intrinsic description of these singularities is desirable.

A common technique to study singularities using birational geometry is to apply certain monoidal transformation to the singularity to extract an exceptional projective divisor over it, and then try to deduce some local information of the singularity from the global information of the exceptional divisor. This approach has been successful in many cases: The study of dual complexes of singularities [KX16, dFKX17], the finiteness of the algebraic fundamental group of a klt singularity [Xu14], the ascending chain condition for log canonical thresholds [dFEM10, HMX14], the study of the normalized volume function on klt singularities [Li17, LX16, LX17], and the theory of log canonical complements [PS01, PS09, Bir16a], among others.

2010 Mathematics Subject Classification. Primary 14E30, Secondary 14B05. 
In this article, we use this approach to study the minimal log discrepancies of klt singularities [Amb99, Sho04]. We aim to explain how a bound on the singularities of a plt blow-up implies finiteness of the possible minimal log discrepancies. More precisely, we say that a plt blow-up $\pi: Y \rightarrow X$ at a point $x$ of a klt pair $(X, \Delta)$ is $\epsilon$-plt if the log discrepancies of the corresponding plt pair $\left(Y, \Delta_{Y}+E\right)$ are either zero or greater than $\epsilon$ (see Definition 2.5). We say that the point $x$ of the klt pair $(X, \Delta)$ admits an $\epsilon$-plt blow-up if there exists $\pi$ as above. For $\epsilon$ a positive real number, adjunction [Hac14, Theorem 0.1] and the boundedness of Fano varieties [Bir16b, Theorem 1.1], allows us to conclude that the normal projective varieties $E$ belong to a bounded family. By [Xu14, Lemma 1], we know that every klt singularity admits a plt blow-up and a simple argument using resolution of singularities proves that every klt singularity admits an $\epsilon$-plt blow-up for some positive real number $\epsilon$ (see Lemma 2.7).

Using the above notation we can introduce the set of minimal log discrepancies of $a$-log canonical pairs admitting an $\epsilon$-plt blow-up:

$$
\mathcal{M}(d, \mathcal{R})_{a, \epsilon}:=\left\{\operatorname{mld}_{x}(X, \Delta) \mid \begin{array}{c}
\operatorname{dim}(X)=d, \text { coeff }(\Delta) \in \mathcal{R}, \text { and }(X, \Delta) \text { is a } a \text {-lc pair } \\
\text { which admits an } \epsilon \text {-plt blow-up at } x
\end{array}\right\} .
$$

We recall the conjecture known as the ascending condition for minimal log discrepancies:

Conjecture 1. Let $d$ be a positive integer and $\mathcal{R}$ be a set of real numbers satisfying the descending chain condition. Then the set $\mathcal{M}(d, \mathcal{R})_{0,0}$ satisfies the ascending chain condition.

The ascending chain condition for minimal log discrepancies is known for surface singularities [Ale93], for certain terminal threefold singularities [Sho96, Lemma 4.4.1], and for toric singularities [Bor97, Amb06]. However, in higher dimensions there is not much that we can say about the possible mld's in a fixed dimension. In this paper, we give a first step towards the understanding of higher dimensional minimal log discrepancies. The following theorem can be understood as a natural geometric stratification of the possible mld's of a fixed dimension by finite sets:

Theorem 1. Let $d$ be a positive integer and let a and $\delta$ be positive real numbers, and $\mathcal{S}$ the set of standard rational numbers. Then the set $\mathcal{M}(d, \mathcal{S})_{a, \epsilon}$ is finite.

The theorem implies the following corollary towards the ascending chain condition.

Corollary 1. The set $\mathcal{M}(d, \mathcal{S})_{0, \epsilon}$ satisfies the ascending chain condition.

In Section 2.5 we give two examples that show that the theorem does not hold if $a$ and $\epsilon$ are not positive. We also prove that $a$-log canonical singularities which admit an $\epsilon$-plt blow-up have bounded Cartier index.

Theorem 2. Let $d$ be a positive integer, and let a and $\epsilon$ be positive real numbers. There exists $p$ only depending on $d, a$ and $\epsilon$ satisfying the following. Let $(X, \Delta)$ be a d-dimensional a-log canonical pair with standard coefficients which admits an $\epsilon$-plt blow-up at $x \in X$. Then $p\left(K_{X}+\Delta\right)$ is a Cartier divisor on a neighborhood of $x \in X$.

Exceptional singularities are those klt singularities for which any log canonical threshold is computed at a unique divisorial valuation [Sho00, Definition 1.5]. The exceptional Du Val surface singularities are the $E_{6}, E_{7}$ and $E_{8}$ singularities. Hypersurfaces exceptional singularities were studied by Ishii and Prokhorov [IP01]. In dimension 3, Prokhorov and Markusevich proved that there are only finitely many $\epsilon$-log canonical exceptional quotient singularities [MP99]. However, a classification of exceptional singularities in higher dimensions seems unfeasable. In this direction, we prove the following application of our main theorem:

Corollary 2. The ascending chain condition for minimal log discrepancies of exceptional singularities with standard coefficients holds. 
It is worth mentioning that Corollary 2 follows almost directly from the proof of [PS01, Theorem 4.4] and [Bir16b, Theorem 1.1]. These two results together with [Ish00] and [Fuj01] are the main motivation of Theorem 1. The proof of [PS01, Theorem 4.4] already contains some of the ideas used in this article.

Acknowledgements. The author would like to thank Caucher Birkar, Christopher Hacon, Harold Blum, Stefano Filipazzi, and Yuri Prokhorov for many useful comments. The author was partially supported by NSF research grants no: DMS-1300750, DMS-1265285 and by a grant from the Simons Foundation; Award Number: 256202. After completing this project, the author was informed that J. Han, J. Liu and V. Shokurov have obtained the results of this paper with more general coefficients [HLS18].

\section{Preliminaries}

All varieties in this paper are quasi-projective over a fixed algebraically closed field of characeristic zero unless stated otherwise. In this section we collect some definitions and preliminary results which will be used in the proof of the main theorem.

2.1. Singularities. In this subsection, we recall the singularities of the minimal model program, the set of standard coefficients and exceptional singularities. We also prove some basic properties about singularities.

Definition 2.1. In this paper a sub-pair $(X, \Delta)$ consists of a normal quasi-projective variety $X$ and a $\mathbb{Q}$ divisor $\Delta$ so that $K_{X}+\Delta$ is a $\mathbb{Q}$-Cartier $\mathbb{Q}$-divisor. If the coefficients of $\Delta$ are non-negative then we say that $(X, \Delta)$ is a $\log$ pair, or simply a pair.

Let $\pi: W \rightarrow X$ be a log resolution of the pair $(X, \Delta)$ and denote by $K_{W}+\Delta_{W}+F_{W}$ the log pull-back of $K_{X}+\Delta$, where $\Delta_{W}$ is the strict transform of $\Delta$ on $W$ and $F_{W}$ is an exceptional divisor. The discrepancy of a prime divisor $E$ on $W$ with respect to the pair $(X, \Delta)$ is

$$
a_{E}(X, \Delta):=-\operatorname{coeff}_{E}\left(\Delta_{W}+F_{W}\right) .
$$

The log discrepancy of a prime divisor $E$ on $W$ with respect to the pair $(X, \Delta)$ is the value

$$
a_{E}(X, \Delta)+1 \text {. }
$$

The center of $E$ on $X$ is its image on $X$ via the morphism $\pi$. We denote by $c_{X}(E)$ the center of the prime divisor $E$ on the variety $X$. We say that the sub-pair $(X, \Delta)$ is sub- $\epsilon$-log canonical if

$$
a_{E}(X, \Delta) \geq-1+\epsilon
$$

for every prime divisor $E$ on $W$. If $(X, \Delta)$ is a pair then we say that is $\epsilon$-log canonical in the above situation. If $\epsilon>0$ is arbitrary we may also say that $(X, \Delta)$ is Kawamata $\log$ terminal (or klt) and if $\epsilon=0$ we just say that the pair is $\log$ canonical, equivalently, the center of a $\log$ canonical place. The total discrepancy $a(X, \Delta)$ of the pair $(X, \Delta)$ is the infimum between all discrepancies $a_{E}(X, \Delta)$ with $E$ a prime divisor over $X$. Thus, $(X, \Delta)$ is $a-\log$ canonical if and only if $a(X, \Delta)+1 \geq a$.

Let $(X, \Delta)$ be a $\log$ canonical pair. A log canonical place of $(X, \Delta)$ is a prime divisor $E$ on a birational model of $X$ so that $a_{E}(X, \Delta)=-1$. A log canonical center of $(X, \Delta)$ is the image on $X$ of a $\log$ canonical place.

Definition 2.2. Let $(X, \Delta)$ be a log pair and $x \in X$. The minimal log discrepancy of $(X, \Delta)$ at $x$ is

$$
\operatorname{mld}_{x}(X, \Delta):=\inf \left\{a_{E}(X, \Delta)+1 \mid E \text { is a prime divisor over } X \text { so that } c_{X}(E)=x\right\} .
$$

If $(X, \Delta)$ is a log canonical pair, taking a log resolution and using [KM98, Lemma 2.29] we can see that the above infimum is indeed a minimum. Observe that $\operatorname{mld}_{x}(X, \Delta) \geq 0$ if and only if the pair $(X, \Delta)$ is $\log$ canonical at $x \in X$. Moreover, $\operatorname{mld}_{x}(X, \Delta)>0$ if and only if the pair $(X, \Delta)$ is klt at $x \in X$. On the other hand, if $(X, \Delta)$ is not $\log$ canonical at $x \in X$, then $\operatorname{mld}_{x}(X, \Delta)=-\infty$ (see, e.g. [KM98, Corollary 2.32]). 
Definition 2.3. We say that the pair $(X, \Delta)$ is divisorial log terminal (or dlt) if the following conditions hold:

(1) there exists a closed subset $Z \subset X$ so that $X \backslash Z$ is smooth,

(2) $\left.\Delta\right|_{X \backslash Z}$ has simple normal crossing support, and

(3) every divisor $E$ over $X$ with center on $Z$ has positive log discrepancy with respect to $(X, \Delta)$.

A pair $(X, \Delta)$ is called purely log terminal (or plt) if the log discrepancy of every exceptional prime divisor over $X$ is strictly positive. In this case $\lfloor\Delta\rfloor$ is a disjoint union of normal prime divisors.

Definition 2.4. Given a finite set $\mathcal{R}$ of rational numbers, we denote by

$$
\mathcal{H}(\mathcal{R}):=\{1\} \cup\left\{1-\frac{r}{m} \mid r \in \mathcal{R} \text { and } m \in \mathbb{Z}_{>0}\right\},
$$

and call $\mathcal{H}(\mathcal{R})$ the set of hyperstandard coefficients associated to $\mathcal{R}$. We denote $\mathcal{S}:=\mathcal{H}(\{1\})$ and call this the set of standard coefficients. In what follows, we will focus on pairs $(X, \Delta)$ so that the coefficients of the boundary $\operatorname{coeff}(\Delta)$ belong to the set of standard coefficients. In this case, we say that the coefficients of $\Delta$ are standard.

Definition 2.5. A plt blow-up of a $\log$ pair $(X, \Delta)$ at a point $x \in X$ is a projective birational morphism $\pi: Y \rightarrow X$ with the following properties:

(1) $Y$ is a quasi-projective normal variety,

(2) the exceptional locus of $\pi$ is an irreducible divisor $E$ whose image on $X$ is $x$,

(3) the pair $\left(Y, \Delta_{Y}+E\right)$ is purely log terminal, where $\Delta_{Y}$ is the strict transform of $\Delta$ on $Y$, and

(4) $-E$ is ample over $X$.

We say that the pair $(X, \Delta)$ admits a plt blow-up at $x \in X$ if there exists $\pi$ with the above conditions. Moreover, we say that the plt blow-up is an $\epsilon$-plt blow-up if any log discrepancy of $\left(Y, \Delta_{Y}+E\right)$ is either zero or greater than $\epsilon$. Analogously, we say that the pair $(X, \Delta)$ admits an $\epsilon$-plt blow-up at $x \in X$ if there exists $\pi$ with the above conditions.

Definition 2.6. The exceptional divisor of a plt blow-up are often called Kollár components of the singularity [LX17, Definition 1.1]. We may use both acceptions on this paper. We may also call a Kollár component over the klt pair $(X, \Delta)$ the log Fano pair

$$
K_{E}+\Delta_{E}:=\left.\left(K_{Y}+\Delta_{Y}+E\right)\right|_{E}
$$

obtained by adjunction to the $E \subset Y$. Observe that $-\left(K_{E}+\Delta_{E}\right)$ is ample and $\left(E, \Delta_{E}\right)$ is klt. Hence, $\left(E, \Delta_{E}\right)$ is a log Fano pair [Bir16a, 2.10].

Proposition 2.7. Let $(X, \Delta)$ be a klt pair and $x \in X$. There exists an $\epsilon$-plt blow-up of $(X, \Delta)$ at $x$ for some positive $\epsilon$.

Proof. By [Xu14, Lemma 1], we can construct a plt blow-up $\pi: Y \rightarrow X$ of $(X, \Delta)$ over $x$. Let $\pi_{Y}: W \rightarrow Y$ be a $\log$ resolution of $\left(Y, \Delta_{Y}+E\right)$, so we can write

$$
K_{W}+\Delta_{W}+F_{W}=\pi_{Y}^{*}\left(K_{Y}+\Delta_{Y}+E\right),
$$

where $\Delta_{W}$ is the strict transform of $\Delta_{Y}$ on $W$, and $F_{W}$ is an exceptional divisor. We can take $\epsilon$ small enough so that

$$
\operatorname{coeff}\left(\Delta_{W}+F_{W}-E_{W}\right)<1-\epsilon
$$

where $E_{W}$ is the strict transform of $E$ on $W$. By [KM98, Lemma 2.29] we conclude that every exceptional divisor over $W$ has $\log$ discrepancy either zero or greater than $\epsilon$ with respect to the purely $\log$ terminal pair $\left(Y, \Delta_{Y}+E\right)$. 
Theorem 1 motivates the following natural invariant of klt singularities.

Definition 2.8. Let $(X, \Delta)$ be a klt pair and $x \in X$. We define the mildest component, or $\mathcal{M C}$ for simplicity, of $(X, \Delta)$ at the point $x \in X$ to be:

$$
\mathcal{M C}_{x}(X, \Delta):=\sup \left\{a\left(E, \Delta_{E}\right)+1 \mid\left(E, \Delta_{E}\right) \text { is a Kollár component of }(X, \Delta) \text { over } x \in X\right\} .
$$

In Proposition 2.21, we will prove that the $\mathcal{M C}$ is indeed attained by some Kollár component over $x \in X$. In this setting, the conditions of Theorem 1 and Theorem 2 can be abbreviated as $(X, \Delta)$ is a $d$-dimensional pair which is $a-\log$ canonical at $x \in X$ and $\mathcal{M C}_{x}(X, \Delta) \geq \epsilon$.

Remark 2.9. In general one could define the mildest component of $(X, \Delta)$ at $x \in X$ for every Grothendieck point of the variety $X$. However, by cutting down with general hyperplanes, the study of minimal log discrepancies on a variety $X$ of dimension $d$ at a point $x \in X$ of codimension $k$ is equivalent to the stuty of minimal $\log$ discrepancies on a variety $X$ of dimension $d-k$ at a closed point.

Definition 2.10. A dlt modification of a $\log$ canonical pair $(X, \Delta)$ is a projective birational morphism $\pi: Y \rightarrow X$ so that $\pi^{*}\left(K_{X}+\Delta\right)=K_{Y}+\Delta_{Y}+E_{Y}$, where $\Delta_{Y}$ is the strict transform of $\Delta$ on $X$ and $E_{Y}$ is an exceptional divisor over $X$ so that the sub-pair $\left(Y, \Delta_{Y}+E_{Y}\right)$ is a dlt pair. It is known that every $\log$ canonical pair admits a dlt modification [KK10, Theorem 3.1].

Definition 2.11. We say that a klt pair $(X, \Delta)$ is exceptional at $x \in X$ if for every boundary $\Gamma \geq 0$ on $X$ so that $(X, \Delta+\Gamma)$ is a $\log$ canonical pair, any dlt modification of $(X, \Delta+\Gamma)$ is indeed plt.

Remark 2.12. It is known that if $(X, \Delta)$ is exceptional at $x$ and $X$ is $\mathbb{Q}$-factorial, then there exists a unique prime divisor over $X$ which compute all $\log$ canonical thresholds over $x \in X$ [MP99, Proposition 2.9].

Lemma 2.13. Let $(X, \Delta)$ be a klt pair which is exceptional at $x \in X$. Assume that the coefficients of $\Delta$ are standard. There exists an $\epsilon_{d}$-blow-up of $(X, \Delta)$ at $x$, for some positive real number $\epsilon_{d}$ which only depends on the dimension $d$ of $X$.

Proof. By Lemma 2.7, we can extract an $\epsilon$-plt blow-up over $x \in X$ for some real number $\epsilon$. By [Bir16a, Theorem 1.8] there exists a strong $n$-complement $K_{Y}+\Delta_{Y}+\Gamma_{Y}+E$ for $K_{Y}+\Delta_{Y}+E$ over $x$, for $n$ only depending on $d$ and $\mathcal{S}$. Since $\mathcal{S}$ is a fixed set then $n$ only depends on $d$. Since $K_{Y}+\Delta_{Y}+\Gamma_{Y}+E$ is $\mathbb{Q}$-linearly trivial over $x \in X$ then we can write

$$
K_{Y}+\Delta_{Y}+\Gamma_{Y}+E=\pi^{*}\left(K_{X}+\Delta+\Gamma\right)
$$

for some boundary $\Gamma$ on $X$ so that $(X, \Delta+\Gamma)$ is a $\log$ canonical pair. By the exceptionality of $(X, \Delta)$ at $x$ we deduce that $(X, \Delta+\Gamma)$ has a unique $\log$ canonical place and hence $\left(Y, \Delta_{Y}+\Gamma_{Y}+E\right)$ has a unique $\log$ canonical place $E$. Moreover, since $n\left(K_{Y}+\Delta_{Y}+\Gamma_{Y}+E\right)$ is Cartier over $x$, we conclude that

$$
a_{F}\left(Y, \Delta_{Y}+E\right)+1 \geq a_{F}\left(Y, \Delta_{Y}+\Gamma_{Y}+E\right)+1 \geq \frac{1}{n}
$$

for every divisor $F$ over $Y$ which is not equal to $E$. Thus, it suffices to take $\epsilon_{d}=\frac{1}{n}$.

2.2. Bounded families. In this subsection, we recall the definition of a log bounded family and prove some properties of such families.

Definition 2.14. We say that a set of pairs $\mathcal{P}$ is $\log$ bounded if there exists a projective morphism $\mathcal{X} \rightarrow T$ of possibly reducible varieties and a divisor $\mathcal{B}$ on $\mathcal{X}$ so that for every pair $(X, \Delta) \in \mathcal{P}$ there exists a closed point $t \in T$ and an isomorphism $\phi: \mathcal{X}_{t} \rightarrow X$ so that $\left(\mathcal{X}_{t}, \mathcal{B}_{t}\right)$ is a pair and $\phi_{*}^{-1} D \leq \mathcal{B}_{t}$. If moreover $\phi$ induces an isomorphism of pairs between $(X, \Delta)$ and $\left(\mathcal{X}_{t}, \mathcal{B}_{t}\right)$, meaning that for every prime divisor $D$ on $X$ we have that

$$
\operatorname{coeff}_{D}(\Delta)=\operatorname{coeff}_{\phi_{*}^{-1} D}\left(\mathcal{B}_{t}\right)
$$


we will say that the set of pairs $\mathcal{P}$ is strictly log bounded. We say that $\mathcal{X} \rightarrow T$ is a bounding family for the varieties $X$ and that $\mathcal{B}$ is a bounding divisor for the set of divisors $\{\Delta \mid(X, \Delta) \in \mathcal{P}\}$.

The following lemma follows from the definition of strictly log bounded family.

Lemma 2.15. Let $\mathcal{P}$ be a $\log$ bounded family of pairs so that the set $\{\operatorname{coeff}(\Delta) \mid(X, \Delta) \in \mathcal{P}\}$ is finite. Then the family $\mathcal{P}$ is strictly log bounded.

Lemma 2.16. Let $\mathcal{P}$ be a bounded family of d-dimensional projective varieties and $\mathcal{Q}$ a set of pairs $\{(X, \Delta) \mid$ $X \in \mathcal{P}\}$, so that coeff $(\Delta)$ satisfies the descending chain condition and for every $(X, \Delta) \in \mathcal{Q}$ we have that $-\left(K_{X}+\Delta\right)$ is pseudo-effective. Then $\mathcal{Q}$ is a log bounded set of pairs.

Proof. We can find a positive constant $C$ so that for each $X \in \mathcal{P}$ there is a very ample Cartier divisor $A$ with $A^{d} \leq C$. Morever, we can assume that $A^{d-1}\left(-K_{X}\right) \leq C$. The set coeff $(\Delta)$ satisfies the descending chain condition, so there exists $\delta>0$ small enough so that $\delta<\operatorname{coeff}(\Delta)$ for every boundary $\Delta$ of a pair $(X, \Delta)$ on $\mathcal{Q}$. Since $-\left(K_{X}+\Delta\right)$ is pseudo-effective we have that $A^{d-1} \cdot\left(-K_{X}+\Delta\right) \geq 0$, hence we conclude that

$$
A^{d-1} \cdot\left(\frac{1}{\delta} \Delta_{\text {red }}\right) \leq A^{d-1} \cdot \Delta \leq A^{d-1} \cdot\left(-K_{X}\right) \leq C,
$$

where $\Delta_{\text {red }}$ is $\Delta$ with the reduced structure. Thus, we get that $A^{d-1} \cdot \Delta_{\text {red }} \leq \delta C$, so by [Ale94, Lemma 3.7.(2)] we conclude that the set of pairs $\mathcal{Q}$ is $\log$ bounded.

Definition 2.17. Let $X$ be an irreducible projective variety of dimension $d$ and let $D$ be a $\mathbb{Q}$-Cartier divisor on $X$. The volume of $D$ is

$$
\operatorname{vol}(D):=\limsup _{m \rightarrow \infty} \frac{d ! h^{0}\left(X, \mathcal{O}_{X}(m D)\right)}{m^{n}} .
$$

In particular, a big divisor has positive volume.

Lemma 2.18. Let $\mathcal{P}$ be a strictly log bounded family of d-dimensional klt pairs. Then there exist positive real numbers $v_{1}$ and $v_{2}$, only depending on $\mathcal{P}$, so that for every $(X, \Delta) \in \mathcal{P}$ with $-\left(K_{X}+\Delta\right)$ ample we have that

$$
v_{1}<\operatorname{vol}\left(-\left(K_{X}+\Delta\right)\right)<v_{2} .
$$

Proof. Since ampleness is open in families, we may restrict to an open set $U \subset T$ so that $-\left(K_{\mathcal{X}_{t}}+\mathcal{B}_{t}\right)$ is ample for every $t \in U$. Furthermore, by Noetherian induction we may assume that the induced morphism $\mathcal{X}_{U} \rightarrow U$ is a projective smooth morphism of relative dimension $d$ with normal fibers. In this case, we have that

$$
\operatorname{vol}\left(-\left(K_{\mathcal{X}_{t}}+\mathcal{B}_{t}\right)\right)=\left(-\left(K_{\mathcal{X}_{t}}+\mathcal{B}_{t}\right)\right)^{d}
$$

is an upper-semicontinuous function on $t \in U$. Hence, it takes finitely many values on $U$.

Lemma 2.19. Let $\mathcal{P}$ be a strictly log bounded family of d-dimensional klt pairs. We can find a positive real number $M$, only depending on $\mathcal{P}$, so that for every $(X, \Delta) \in \mathcal{P}$ with $-\left(K_{X}+\Delta\right)$ ample there exists an ample curve $C$ on $X$ so that

$$
-\left(K_{X}+\Delta\right) \cdot C \leq M
$$

Proof. Since the family of pairs is strictly log bounded, we may find a positive natural number $m$, only depending on $\mathcal{P}$, so that $\left|-m\left(K_{X}+\Delta\right)\right|$ is a base point free linear system for any $(X, \Delta)$ as in the statement. Hence, a general curve $C$ on the rational equivalence class of $\left(-m\left(K_{X}+\Delta\right)\right)^{d-1}$ will be an ample curve. Moreover, by Lemma 2.18, we have that

$$
-\left(K_{X}+\Delta\right) \cdot C=m^{d-1}\left(-\left(K_{X}+\Delta\right)\right)^{d}=m^{d-1} \operatorname{vol}\left(-\left(K_{X}+\Delta\right)\right)<m^{d-1} v_{2} .
$$

Thus, it suffices to take $M=m^{d-1} v_{2}$. 
Lemma 2.20. Let $d$ be a positive integer and $\epsilon$ a positive real number. There exists a constant $l$ only depending on $d$ and $\epsilon$ satisfying the following. Let $X$ be a d-dimensional klt variety so that $K_{X}$ is Cartier at $x \in X$. Assume that $X$ admits an $\epsilon$-plt blow-up at $x \in X$ extracting a divisor $E$. Then we have that

$$
a_{E}(X, 0) \leq l .
$$

In particular, there are finitely many possible values for $a_{E}(X, 0)$.

Proof. Let $\pi: Y \rightarrow X$ be the $\epsilon$-plt blow-up at $x \in X$ and write

$$
K_{Y}-a_{E}(X, 0) E=\pi^{*} K_{X} .
$$

By assumption of $X$ being klt and $K_{X}$ being Cartier at $x$ we know that $a_{E}(X, 0)$ is a non-negative integer. We write

$$
K_{E}+\Delta_{E}=\left.\left(K_{Y}+E\right)\right|_{E} .
$$

Thus, the pair $\left(E, \Delta_{E}\right)$ is $\epsilon$-lc and $-\left(K_{E}+\Delta_{E}\right)$ is ample. By boundedness of Fano varieties [Bir16b, Theorem 1.1] we conclude that the projective varieties $E$ belong to a bounded family. By [HK10, Theorem 3.34] we know that the coefficients of $\Delta_{E}$ are standard, therefore the pairs $\left(E, \Delta_{E}\right)$ are log bounded by Lemma 2.16. Moreover, since $\left(E, \Delta_{E}\right)$ is $\epsilon$-log canonical then the coefficients of $\Delta_{E}$ are at most $1-\epsilon$, hence belong to a finite set of rational numbers. By Lemma 2.15, conclude that the pairs $\left(E, \Delta_{E}\right)$ belong to a strictly $\log$ bounded family $\mathcal{P}$, which only depends on the dimension $d$ and the positive real number $\epsilon$.

By [Sho93, Proposition 3.9], we know that at codimension 2 points of $Y$, every Weil divisor has Cartier index bounded by $p$, for some constant $p$ which only depends on $\epsilon$. Hence, there exists a closed subset $Z$ on $Y$ of codimension at least 3, so that the Cartier index of $E$ is a divisor of $p$ outside $Z$. By Lemma 2.19, we may find an ample curve $C$ so that

$$
-\left(K_{E}+\Delta_{E}\right) \cdot C \leq M
$$

for some constant $M$ which only depends on $\mathcal{P}$. Thus, up to replacing $C$ with a rationally equivalent curve we may assume that $C$ does not intersect $Z$, so we have $p E \cdot C$ is a negative integer, or equivalently,

$$
-E \cdot C \in \mathbb{Z}_{>0}\left[\frac{1}{p}\right] .
$$

Moreover, since $\left(E, \Delta_{E}\right)$ belongs to a strictly log bounded family we conclude that the Cartier index of $-\left(K_{E}+\Delta_{E}\right)$ is bounded by a constant which only depends on $\mathcal{P}$ [Bir16a, Lemma 2.25]. In particular,

$$
-\left(K_{Y}+E\right) \cdot C=-\left(K_{E}+\Delta_{E}\right) \cdot C
$$

belongs to a finite set $\mathcal{F}$ of positive rational numbers, which only depends on $\mathcal{P}$. Finally, observe that from the relation

we conclude that

$$
\left(K_{Y}+E-\left(a_{E}(X, 0)+1\right) E\right) \cdot C=\pi^{*}\left(K_{X}\right) \cdot C=0,
$$

so

$$
\frac{\left(a_{E}(X, 0)+1\right)}{p} \leq\left(a_{E}(X, 0)+1\right)(-E \cdot C) \in \mathcal{F},
$$

$$
a_{E}(X, 0) \leq p \max \{\mathcal{F}\}-1,
$$

where the right hand side only depends on $\epsilon$ and $\mathcal{F}$. Since $\mathcal{F}$ only depends on $\mathcal{P}$, and $\mathcal{P}$ only depends on $d$ and $\epsilon$, we deduce that $l=p \max \{\mathcal{F}\}-1$ only depends on $d$ and $\epsilon$. This proves the first statement. Since $a_{E}(X, 0)$ is a non-negative integer, we conclude that there are finitely many possible values for it, proving the second statement.

To conclude this subsection, we will prove that the mildest component of a klt singularity is indeed attained by a Kollár component, or equivalently, the infimum in Definition 2.8 is a minimum. 
Proposition 2.21. Let $(X, \Delta)$ be a klt singularity so that the coefficients of $\Delta$ are rational numbers and $x \in X$. There exists a Kollár component $\pi: Y \rightarrow X$ extracting a divisor $E \subset Y$ so that

$$
\mathcal{M C}_{x}(X, \Delta)=a\left(Y, \Delta_{Y}+E\right) .
$$

Proof. We proceed by contradiction. Assume we have a sequence of Kollár components $\pi_{i}: Y_{i} \rightarrow X$ over $x \in X$ for the pair $(X, \Delta)$ so that the total discrepancies of the pairs $\left(E_{i}, \Delta_{E_{i}}\right)$ are in an infinite increasing sequence. Since the coefficients of $\Delta_{Y_{i}}$ are fixed by coeff $(\Delta)$, we conclude that the coefficients of $\Delta_{E_{i}}$ belong to a finite set of rational numbers which only depend on coeff $(\Delta)$ [FM18, Lemma 5.3]. By [Bir16a, Theorem 1.1], Lemma 2.16, and Lemma 2.15 we conclude that the $\log$ pairs $\left(E_{i}, \Delta_{E_{i}}\right)$ belong to a strictly $\log$ bounded family. This contradicts the fact that the total log discrepancy can take only finitely many values on bounded families.

2.3. Finite morphisms. In this subsection, we recall the index one cover of a log canonical singularity and the behaviour of log discrepancies under finite dominant morphisms.

Definition 2.22. Let $(X, \Delta)$ be a pair and write $\Delta=\sum_{i} d_{i} \Delta_{i}$ where the $\Delta_{i}$ 's are pairwise different prime divisors on $X$. Given a quasi-finite morphism $\phi: X^{\prime} \rightarrow X$ between normal varieties, we can write

$$
\phi^{*}\left(K_{X}+\Delta\right)=K_{X^{\prime}}+\Delta^{\prime},
$$

where

$$
\Delta^{\prime}:=\sum_{i} \sum_{f\left(E_{j}\right)=\Delta_{i}}\left(d_{i}\left(r_{j}+1\right)-r_{j}\right) E_{j}
$$

and $r_{j}$ is the ramification index at the generic point of $E_{j}$ [Sho93, 2.1]. The above formula is called the pull-back formula for quasi-finite morphisms.

The following lemma follows from the pull-back formula for quasi-finite morphisms.

Lemma 2.23. Let $(X, \Delta)$ be a pair with standard coefficients one and $\phi: X^{\prime} \rightarrow X$ be a finite morphism of normal varieties. Assume that for every prime divisor $E$ on $X^{\prime}$ we have that $r_{E}+1$ divides $\left(1-d_{i}\right)^{-1}$, where $r_{E}$ is the ramification index of $\phi$ at $E$ and $d_{i}$ is the coefficient of $\phi(E)$ at $\Delta$. Then $\Delta^{\prime}$ is an effective divisor whose coefficients are standard.

The following is a theorem of Zariski that is often used instead of resolution of singularities [Zar39].

Theorem 2.24. Let $Y^{\prime}$ and $X$ be two integral schemes of finite type over a field over $\mathbb{Z}$, and $f: Y^{\prime} \rightarrow X$ a dominant morphism. Let $D \subset Y^{\prime}$ be a prime divisor and $\eta \in D$ the generic point. Assume that $Y^{\prime}$ is normal at $\eta$. We can define a sequence of schemes and rational maps as follows:

(1) $X_{0}=X$ and $f_{0}=f$

(2) If $f_{i}: Y^{\prime} \rightarrow X_{i}$ is defined, then let $Z_{i} \subset X_{i}$ the closure of $f_{i}(\eta)$. We define $X_{i+1}$ to be the blow-up of $X_{i}$ at $Z_{i}$ and $f_{i+1}: Y^{\prime} \rightarrow X_{i+1}$ the induced rational map.

For $j$ large enough $\operatorname{dim}\left(Z_{j}\right) \geq \operatorname{dim}(X)-1$ and $X_{j}$ is regular at the generic point of $Z_{j}$.

Lemma 2.25. With the assumptions of Lemma 2.23. The following conditions hold:

(1) For $\epsilon$ a non-negative real number the pair $\left(X^{\prime}, \Delta^{\prime}\right)$ is $\epsilon$-log canonical if and only if $(X, \Delta)$ is $\epsilon$-log canonical, and

(2) if $(X, \Delta)$ is a log canonical pair with a unique log canonical center $x \in X,(X, \Delta)$ has a unique $\log$ canonical place, and $x^{\prime}=\phi^{-1}(x)$ is a point, then $\left(X^{\prime}, \Delta^{\prime}\right)$ has a unique log canonical place. 
Proof. Let $\pi: Y \rightarrow X$ be a projective birational morphism and $Y^{\prime} \rightarrow Y \times_{X} X^{\prime}$ the normalization of the main component of the fiber product, then we have a commutative diagram

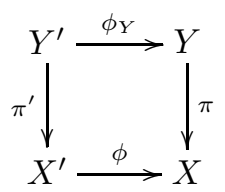

and we can write

$$
\pi^{\prime *}\left(K_{X^{\prime}}+\Delta^{\prime}\right)=K_{Y^{\prime}}+\Delta_{Y^{\prime}}^{\prime}+E^{\prime} \text { and } \pi^{*}\left(K_{X}+\Delta\right)=K_{Y}+\Delta_{Y}+E .
$$

Here $\Delta_{Y^{\prime}}^{\prime}$ is the strict transform of $\Delta^{\prime}$ on $Y^{\prime}$. By Lemma 2.23, we know that $\left(X^{\prime}, \Delta^{\prime}\right)$ is a log pair. Thus, we have the relation

$$
\phi_{Y}^{*}\left(K_{Y}+\Delta_{Y}+E\right)=K_{Y^{\prime}}+\Delta_{Y^{\prime}}^{\prime}+E^{\prime} .
$$

Therefore, by the pull-back formula for quasi-finite morphisms we have that

$$
a_{E}\left(X^{\prime}, \Delta^{\prime}\right)+1=r_{E}\left(a_{E}(X, \Delta)+1\right)
$$

where $r_{E}$ is the ramification index of $\phi_{Y}$ at the generic point of $E$. By Theorem 2.24, we know that for every divisorial valuation on $X^{\prime}$ we can find $\pi: Y \rightarrow X$ so that the center of this valuation on $Y^{\prime}$ is a divisor, where $Y^{\prime}$ is as in the commutative diagram (2.1). This proves the first statement.

Now we turn to prove the second statement by contradiction. By the proof of the first statement we know that the log canonical pair $\left(X^{\prime}, \Delta^{\prime}\right)$ has at least one log canonical place, and all its log canonical places map onto $x^{\prime} \in X^{\prime}$. Assume that $\left(X^{\prime}, \Delta^{\prime}\right)$ has more than one $\log$ canonical place. Let $\pi: Y^{\prime} \rightarrow X^{\prime}$ be a dlt modification of $\left(X^{\prime}, \Delta^{\prime}\right)$ and write

$$
\pi^{\prime *}\left(K_{X^{\prime}}+\Delta^{\prime}\right)=K_{Y^{\prime}}+\Delta_{Y^{\prime}}^{\prime}
$$

Applying [KM98, Theorem 5.48] to a log resolution of $\left(Y^{\prime}, \Delta_{Y}^{\prime}\right)$ we deduce that $\left\lfloor\Delta_{Y^{\prime}}^{\prime}\right\rfloor$ is connected. Moreover, since $\left(X^{\prime}, \Delta^{\prime}\right)$ has more than one log canonical place, the divisor $\left\lfloor\Delta_{Y^{\prime}}^{\prime}\right\rfloor$ is connected and has at least two irreducible components. Since the intersection of two $\log$ canonical centers is a union of $\log$ canonical centers [Amb11, Theorem 1.1], we deduce that $\left(Y^{\prime}, \Delta_{Y^{\prime}}^{\prime}\right)$ has infinitely many log canonical places. Thus, $\left(X^{\prime}, \Delta^{\prime}\right)$ has infinitely many log canonical places. By Theorem 2.24, we conclude that each of those $\log$ canonical places appears in a commutative diagram as in (2.1). Moreover, at least one log canonical places of $\left(X^{\prime}, \Delta^{\prime}\right)$ is exceptional over the dlt model $\left(Y, \Delta_{Y}\right)$ of $(X, \Delta)$. Therefore, $(X, \Delta)$ has at least two $\log$ canonical places. This provides the needed contradiction and the claim follows.

Definition 2.26. Let $(X, \Delta)$ be a klt pair with standard coefficients and $x \in X$ a point. Consider $a$ the smallest positive integer so that $a\left(K_{X}+\Delta\right) \sim 0$ on a neighborhod of $x \in X$, or equivalently, $a$ is the Cartier index of $K_{X}+\Delta$ at $x \in X$. Therefore we have an isomorphism $\mathcal{O}_{X}\left(a\left(K_{X}+\Delta\right)\right) \simeq \mathcal{O}_{X}$, we can choose a nowhere zero section

$$
s \in H^{0}\left(X, \mathcal{O}_{X}\left(a\left(K_{X}+\Delta\right)\right)\right),
$$

and consider $\phi: X^{\prime} \rightarrow X$ the corresponding cyclic cover. The ramification index of $\phi$ at a prime divisor $E$ which maps onto $\Delta_{i}$ is exactly $d_{i}-1$, where $d_{i}=\operatorname{coeff}_{\Delta_{i}}(\Delta)$. Moreover, $\phi$ is ramified only at the support of $\Delta$. Therefore, from the pull-back formula for quasi-finite morphisms we know that

$$
K_{X^{\prime}}=\phi^{*}\left(K_{X}+\Delta\right) \text {. }
$$

We call $\phi$ the index one cover of the klt pair $(X, \Delta)$ locally at $x \in X$ [Fuj01, Notation 4.1]. 
2.4. Complements. In this subsection, we prove that klt singularities with plt blow-ups admits local complements with a unique log canonical place.

Definition 2.27. Let $(X, \Delta)$ be a $\log$ canonical pair and $X \rightarrow Z$ be a contraction of normal quasi-projective varieties. We say that $\Gamma \geq 0$ is a strong $(\delta, n)$-complement over $z \in Z$ of $(X, \Delta)$ if the following conditions hold:

- $(X, \Delta+\Gamma)$ is an $\delta$-log canonical pair, and

- $n\left(K_{X}+\Delta+\Gamma\right) \sim 0$ over a neighborhood of $z$.

In the case that $X \rightarrow Z$ is the identity, then we say that $\Gamma$ is a local strong $(\delta, n)$-complement around $x \in X$ for $(X, \Delta)$. On the other hand, if $Z=\operatorname{Spec}(k)$ for some field $k$, then we say that $\Gamma$ is a global strong $(\delta, n)$-complement for the pair $(X, \Delta)$.

Remark 2.28. In this notation, a pair $(X, \Delta)$ is exceptional at $x \in X$ if and only if every local complement at $x \in X$ has a unique log canonical place.

Lemma 2.29. Let $(X, \Delta)$ be a d-dimensional klt pair with standard coefficients. Assume that $(X, \Delta)$ admits an $\epsilon$-plt blow-up at $x \in X$ extracting the exceptional divisor $E$. There exists a natural number $n$, only depending on $d$ and $\epsilon$, and a boundary $\Gamma$ on $X$ so that the following conditions hold:

- $n\left(K_{X}+\Delta+\Gamma\right) \sim 0$ on a neighborhood of $x \in X$, and

- $K_{Y}+\Delta_{Y}+\Gamma_{Y}+E=\pi^{*}\left(K_{X}+\Delta+\Gamma\right)$ is an $\epsilon$-plt pair on a neighborhood of $x \in X$.

Moreover, we may assume that the boundary divisors $\Gamma$ and $\Delta$ do not share prime components.

Proof. We will construct a strong $(0, n)$-complement for the divisor $-\left(K_{Y}+\Delta_{Y}+E\right)$ with respect to the morphism $\pi: Y \rightarrow X$ around $x \in X$. This complement will push-forward to a $(0, n)$-complement for $(X, \Delta)$ locally around $x \in X$. In order to do so, we will do adjunction to $E$, produce a global complement on $E$ and then pull-back to a neighborhood of $E$.

By adjunction, we can write

$$
\left.\left(K_{Y}+\Delta_{Y}+E\right)\right|_{E}=K_{E}+\Delta_{E}
$$

is an $\epsilon$-log canonical pair and the coefficients of $\Delta_{E}$ belong to a set of rational numbers satisfying the descending chain condition with rational accumulation points (see, e.g. [FM18, Lemma 5.3]). Hence, by [FM18, Theorem 1.3.2], we can find $n$ only depending on $d$ and $\epsilon$, and a global strong $(\epsilon, n)$-complement $\Gamma_{E}$ for the pair $\left(E, \Delta_{E}\right)$. From the proof of [FM18, Theorem 1.3] we may assume that $\Gamma_{E}$ do not share prime components with $\Delta_{E}$. Without loss of generality we may assume that $n \Delta_{Y}$ is a Weil divisor.

Let $\pi_{Y}: W \rightarrow Y$ be a $\log$ resolution of $\left(Y, \Delta_{Y}+E\right)$, and write

$$
-N_{W}:=\pi_{Y}^{*}\left(K_{Y}+\Delta_{Y}+E\right)=K_{W}+\Delta_{W}+E_{W},
$$

where $E_{W}$ is the strict transform of $E$ on $W$. We define

$$
L_{W}:=-n K_{W}-n E_{W}-\left\lfloor(n+1) \Delta_{W}\right\rfloor .
$$

Let $P_{W}$ be the unique integral effective divisor on $W$ so that

$$
\Lambda_{W}:=(n+1) \Delta_{W}-\left\lfloor(n+1) \Delta_{W}\right\rfloor+P
$$

is a boundary on $W$ so that $\left(W, \Lambda_{W}\right)$ is plt and $\left\lfloor\Lambda_{W}\right\rfloor=E_{W}$. We claim that $P_{W}$ is an exceptional divisor over $Y$. Indeed, if $D$ is a prime divisor on $W$ which is not contracted on $Y$ then we have that

$$
\operatorname{coeff}_{D}\left(\left\lfloor(n+1) \Delta_{W}\right\rfloor\right)=\operatorname{coeff}_{D}\left(n \Delta_{W}\right)
$$

because $n \Delta_{Y}$ is integral. Therefore

$$
\operatorname{coeff}_{D}\left((n+1) \Delta_{W}-\left\lfloor(n+1) \Delta_{W}\right\rfloor\right)=\operatorname{coeff}_{D}\left(\Delta_{W}\right)=\operatorname{coeff}_{\pi_{Y}(D)}\left(\Delta_{Y}\right) \in(0,1) .
$$


By definition, we have that

$$
L_{W}+P_{W}-E_{W}=K_{W}+\Lambda_{W}-E_{W}+(n+1) N_{W},
$$

is the sum of the klt pair $K_{W}+\Lambda_{W}-E_{W}$ and the nef and big divisor $(n+1) N_{W}$ over a neighborhood of $x \in X$. Shrinking around $x \in X$ we may assume that $X$ is affine, then $(n+1) N_{W}$ is nef and big over $X$. By the relative version of Kawamata-Viehweg theorem [KMM87, Theorem 1-2-5], we have a surjection

$$
H^{0}\left(L_{W}+P_{W}\right) \rightarrow H^{0}\left(\left.\left(L_{W}+P_{W}\right)\right|_{E_{W}}\right) .
$$

We denote by $\Gamma_{E_{W}}$ the pull-back of $\Gamma_{E}$ to $E_{W}$. Observe that we have

$$
\left.\left(L_{W}+P_{W}\right)\right|_{E_{W}} \sim G_{E_{W}}:=n \Gamma_{E_{W}}+n \Delta_{E_{W}}-\left\lfloor(n+1) \Delta_{E_{W}}\right\rfloor+P_{E_{W}},
$$

where $P_{E_{W}}:=\left.P_{W}\right|_{E_{W}}$ and $\Delta_{E_{W}}:=\left.\Delta_{W}\right|_{E_{W}}$. The divisor $G_{E_{W}}$ is integral and its coefficients are strictly greater than -1 , therefore it is indeed effective. By the surjectivity of (2.2) there exists $0 \leq G_{W} \sim L_{W}+P_{W}$ which restricts to $G_{E_{W}}$. We denote by $G_{Y}$ the push-forward of $G_{W}$ to $Y$. By pushing-forward the linear equivalence $L_{W}+P_{W} \sim G_{W}$ to $Y$, and using the fact that $P_{W}$ is $Y$-exceptional we get that

$$
0 \leq G_{Y} \sim-n\left(K_{Y}+\Delta_{Y}+E\right) .
$$

We define $\Gamma_{Y}:=\frac{G_{Y}}{n}$. Observe that by construction we have

$$
n\left(K_{Y}+\Delta_{Y}+\Gamma_{Y}+E\right) \sim 0
$$

on a neighborhood of $x \in X$. We claim that $\left.\Gamma_{Y}\right|_{E}=\Gamma_{E}$. Indeed, observe that we can define

$$
n \Gamma_{W}:=G_{W}-P_{W}+\left\lfloor(n+1) \Delta_{W}\right\rfloor-n \Delta_{W} \sim n N_{W} \sim_{\mathbb{Q}, Y} 0,
$$

and $n \Gamma_{W}$ pushes-forward to $G_{Y}$ on $Y$, hence $\Gamma_{W}=\pi_{Y}^{*}\left(\Gamma_{Y}\right)$. On the other hand, we have $n \Gamma_{E_{W}}=\left.n \Gamma_{W}\right|_{E_{W}}$, which means that $\Gamma_{E_{W}}=\left.\Gamma_{W}\right|_{E_{W}}$. Thus, we have $\left.\Gamma_{Y}\right|_{E}=\Gamma_{E}$ as claimed.

Finally, observe that

$$
\left.\left(K_{Y}+\Delta_{Y}+\Gamma_{Y}+E\right)\right|_{E}=K_{W}+\Delta_{E}+\Gamma_{E},
$$

so by inversion of adjunction we conclude that $\left(Y, \Delta_{Y}+\Gamma_{Y}+E\right)$ is $\epsilon$-plt. Moreover, since $\Gamma_{E}$ and $\Delta_{E}$ do not share prime components, then $\Delta_{Y}$ and $\Gamma_{Y}$ do not share prime components as well. Define $\Gamma=\pi_{*}\left(\Gamma_{Y}\right)$, and observe that

$$
\pi^{*}\left(K_{X}+\Delta+\Gamma\right)=K_{Y}+\Delta_{Y}+\Gamma_{Y}+E .
$$

Thus, $(X, \Delta+\Gamma)$ is a $\log$ canonical pair with a unique $\log$ canonical place so that $n\left(K_{X}+\Delta+\Gamma\right) \sim 0$ on a neighborhood of $x \in X$. Moreover, $\Delta$ and $\Gamma$ do not share prime components.

2.5. Examples. In this subsection, we give two examples to show that the $a$-log canonical and $\epsilon$-plt blow-up conditions of Theorem 1 and Theorem 2 are indeed necessary.

Example 2.30. Let $X_{n}$ be the cone over a rational curve of degree $n$. Blowing-up the vertex $\pi_{n}: Y_{n} \rightarrow X_{n}$ gives a $\log$ resolution so that the pair $\left(Y_{n}, E_{n}\right)$ is $\log$ smooth. Hence, $\pi_{n}$ is a 1-plt blow-up. However, $a_{E_{n}}\left(X_{n}, 0\right)=-1+\frac{2}{n}$, and the Cartier index of $X_{n}$ at the vertex depends on $n$.

Example 2.31. By [Hay99, Proposition 5.1] we can construct terminal threefold singularities $X_{m}$ of index $m$ and extract two different divisors with discrepancies $1 / m$ and $2 / m$, respectively. Hence, if there is any plt blow-up of $X_{m}$ it is an $\epsilon$-plt blow-up for some $\epsilon \leq 2 / m$. 


\section{Proof of the Main Theorem}

Proof of Theorem 1. Let $(X, \Delta)$ be a log pair which admits an $\epsilon$-plt blow-up $\pi: Y \rightarrow X$ at $x \in X$. By assumption $(X, \Delta)$ is $a$-lc at $x \in X$. In particular, the minimal log discrepancy $\operatorname{mld}_{x}(X, \Delta)$ is strictly positive.

Let $\phi: X^{\prime} \rightarrow X$ be the index one cover of the klt pair $(X, \Delta)$ locally at $x$ so that $\phi^{*}\left(K_{X}+\Delta\right)=K_{X^{\prime}}$ (see [Fuj01, Notation 4.1] or Definition 2.26). We denote by $Y^{\prime}$ the normalization of the main component of $X^{\prime} \times_{X} Y$. Hence, we have a commutative diagram

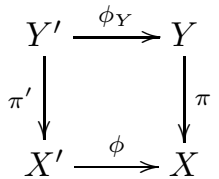

where $\pi^{\prime}$ is birational and $\phi_{Y}$ is finite with the same degree as $\phi$. We can write

$$
\phi^{*}\left(K_{X}+\Delta\right)=K_{X^{\prime}} \quad \text { and } \quad \phi_{Y}^{*}\left(K_{Y}+\Delta_{Y}+E\right)=K_{Y^{\prime}}+E^{\prime}
$$

where $E^{\prime}$ is the reduced exceptional divisor contracted by $\pi^{\prime}$. We claim that the pair $\left(Y^{\prime}, E^{\prime}\right)$ is $\epsilon$-plt. Indeed, by Lemma 2.29 we may find an effective divisor $\Gamma$ on $X$ so that

$$
\pi^{*}\left(K_{X}+\Delta+\Gamma\right)=K_{Y}+\Delta_{Y}+\Gamma_{Y}+E
$$

is an $\epsilon$-plt pair, where $\Gamma_{Y}$ is the strict transform of $\Gamma$ on $Y$. Moreover, $(X, \Delta+\Gamma)$ has a unique log canonical place which corresponds to $E$, and the divisors $\Delta$ and $\Gamma$ do not share prime components. Therefore, by Lemma 2.23 and Lemma 2.25, we conclude that

$$
K_{X^{\prime}}+\Gamma_{X^{\prime}}=\phi^{*}\left(K_{X}+\Delta+\Gamma\right)
$$

is indeed a pair which has a unique log canonical place and its log discrepancies are either zero or greater than $\epsilon$. By the commutativy of the diagram, we have that

$$
\pi^{\prime *}\left(K_{X^{\prime}}+\Gamma_{X^{\prime}}\right)=K_{Y^{\prime}}+\Gamma_{Y^{\prime}}+E^{\prime}
$$

is an $\epsilon$-plt pair. Hence $\left(Y^{\prime}, E^{\prime}\right)$ is $\epsilon$-plt as well. In particular, $\pi^{\prime}: Y^{\prime} \rightarrow X^{\prime}$ is an $\epsilon$-plt blow-up at $x^{\prime} \in X^{\prime}$.

Observe that by construction the $\mathbb{Q}$-divisors $K_{Y}+\Delta_{Y}+E$ and $K_{Y^{\prime}}+E^{\prime}$ are both $\epsilon$-plt and anti-ample over $X$ and $X^{\prime}$ respectively. We define the following pairs by adjunction:

$$
K_{E^{\prime}}+\Delta_{E^{\prime}}=\left.\left(K_{Y^{\prime}}+E^{\prime}\right)\right|_{E^{\prime}} \text { and } K_{E}+\Delta_{E}=\left.\left(K_{Y}+\Delta_{Y}+E\right)\right|_{E} .
$$

By the above considerations, we know that both pairs are anti-ample and $\epsilon$-lc. By [Bir16b, Theorem 1.1], we know that the algebraic varieties $E$ and $E^{\prime}$ belong to a bounded family which only depends on $d-1$ and $\epsilon$. Moreover, the boundary divisors $\Delta_{E}$ and $\Delta_{E^{\prime}}$ have coefficients that belong to a set with the descending chain condition. By Lemma 2.16, we conclude that the $\log$ pairs $\left(E, \Delta_{E}\right)$ and $\left(E^{\prime}, \Delta_{E^{\prime}}\right)$ belong to a $\log$ bounded family which only depends on $d-1, \epsilon$, and the derived set of standard coefficients. Furthermore, by [Bir16a, Lemma 3.3], we know that the coefficients of $\Delta_{E}$ and $\Delta_{E^{\prime}}$ belong to a set of hyperstandard coefficients $\mathcal{H}(\mathcal{R})$ corresponding to a finite set of rational numbers $\mathcal{R}$, which only depends on $\mathcal{S}$. By the $\epsilon$ - $\log$ canonical condition of the pairs $\left(E, \Delta_{E}\right)$ and $\left(E^{\prime}, \Delta_{E^{\prime}}\right)$ and the fact that the only accumulation point of $\mathcal{H}(\mathcal{R})$ is 1 , we conclude that $\Delta_{E}$ and $\Delta_{E^{\prime}}$ have coefficients in the finite set $\mathcal{H}(\mathcal{R}) \cap[0,1-\epsilon)$ which only depends on $\epsilon$. By Lemma 2.15, we deduce that the pairs $\left(E, \Delta_{E}\right)$ and $\left(E^{\prime}, \Delta_{E^{\prime}}\right)$ belong to a strictly log bounded family. Denote by $\phi_{E}$ the restriction of $\phi_{Y}$ to $E^{\prime}$ and observe that

$$
\operatorname{deg}(\phi)=\operatorname{deg}\left(\phi_{Y}\right)=\operatorname{deg}\left(\phi_{E}\right) r_{E},
$$

where $r_{E}$ denotes the ramification index of $\phi_{Y}$ at the generic point of $E^{\prime}$. 
Now we turn to prove that $\operatorname{deg}(\phi)$ has an upper bound which only depends on $d, a$ and $\epsilon$. In order to do so, we just need to provide that there is an upper bound for $\phi_{E}$ and $r_{E}$. Observe that we have

$$
\operatorname{deg}\left(\phi_{E}\right)=\frac{\operatorname{vol}\left(-\left(K_{E^{\prime}}+\Delta_{E^{\prime}}\right)\right)}{\operatorname{vol}\left(-\left(K_{E}+\Delta_{E}\right)\right)} .
$$

Therefore, by Lemma 2.18, there is an upper bound for $\operatorname{deg}\left(\phi_{E}\right)$, which only depends on $d-1$ and $\epsilon$. On the other hand, we have the relation

$$
a_{E^{\prime}}\left(X^{\prime}, 0\right)+1=r_{E}\left(a_{E}(X, \Delta)+1\right) \geq r_{E} a .
$$

By Lemma 2.20 we know that $a_{E^{\prime}}\left(X^{\prime}, 0\right)+1$ has an upper bound which only depends on $d$ and $\epsilon$. We conclude that $\operatorname{deg}(\phi)$ has an upper bound which only depends on $d, a$ and $\epsilon$.

Thus, $\operatorname{mld}_{x}(X, \Delta)$ belongs to a discrete set which only depends on $d, a$ and $\epsilon$. Finally, by Lemma 2.25 we have that

$$
\operatorname{deg}(\phi) \operatorname{mld}_{x}(X, \Delta) \leq \operatorname{mld}_{x^{\prime}}\left(X^{\prime}, 0\right) \leq a_{E^{\prime}}\left(X^{\prime}, 0\right)+1,
$$

therefore $\operatorname{mld}_{x}(X, \Delta)$ belongs to a finite set which only depends on $d, a$ and $\epsilon$.

Proof of Theorem 2. It follows from the bound on $\operatorname{deg}(\phi)$ given in the proof of Theorem 1.

Proof of Corollary 1. If there exists a sequence in $\mathcal{M}(d, \mathcal{S})_{0, \epsilon}$ which contradicts the ascending chain condition, passing to a subsequence we may assume the sequence is strictly increasing. Therefore, such infinite sequence belongs to $\mathcal{M}(d, \mathcal{S})_{a, \epsilon}$ for some positive real number $a$. This contradicts Theorem 1.

Proof of Corollary 2. The proof follows from Theorem 1 and Lemma 2.13.

\section{REFERENCES}

[Ale93] Valery Alexeev, Two two-dimensional terminations, Duke Math. J. 69 (1993), no. 3, 527-545, DOI 10.1215/S00127094-93-06922-0. MR1208810 个2

[Ale94] _, Boundedness and $K^{2}$ for log surfaces, Internat. J. Math. 5 (1994), no. 6, 779-810, DOI 10.1142/S0129167X94000395. MR1298994 $\uparrow 6$

[Amb99] Florin Ambro, On minimal log discrepancies, Math. Res. Lett. 6 (1999), no. 5-6, 573-580, DOI 10.4310/MRL.1999.v6.n5.a10. MR1739216 $\uparrow 2$

[Amb06] $\ldots$, The set of toric minimal log discrepancies, Cent. Eur. J. Math. 4 (2006), no. 3, 358-370, DOI 10.2478/s11533006-0013-x. MR2233855 $\uparrow 2$

[Amb11] _ Basic properties of log canonical centers, Classification of algebraic varieties, EMS Ser. Congr. Rep., Eur. Math. Soc., Zürich, 2011, pp. 39-48, DOI 10.4171/007-1/2. MR2779466 ^9

[Bir16a] Caucher Birkar, Anti-pluricanonical systems on Fano varieties, 2016. https://arxiv.org/abs/1603.05765v3. $\uparrow 1,4,5$, $7,8,12$

[Bir16b] https://arxiv.org/abs/1609.05543v1. $\uparrow 2,3,7,12$

[Bor97] Alexandr Borisov, Minimal discrepancies of toric singularities, Manuscripta Math. 92 (1997), no. 1, 33-45, DOI 10.1007/BF02678179. MR1427666 $\uparrow 2$

[dFEM10] Tommaso de Fernex, Lawrence Ein, and Mircea Mustaţă, Shokurov's ACC conjecture for log canonical thresholds on smooth varieties, Duke Math. J. 152 (2010), no. 1, 93-114, DOI 10.1215/00127094-2010-008. MR2643057 个1

[dFKX17] Tommaso de Fernex, János Kollár, and Chenyang Xu, The dual complex of singularities, Higher dimensional algebraic geometry - in honour of Professor Yujiro Kawamata's sixtieth birthday, Adv. Stud. Pure Math., vol. 74, Math. Soc. Japan, Tokyo, 2017, pp. 103-129. MR3791210 ^1

[Fuj01] Osamu Fujino, The indices of log canonical singularities, Amer. J. Math. 123 (2001), no. 2, 229-253. MR1828222 $\uparrow 3,9,12$

[FM18] Stefano Filipazzi and Joaquín Moraga, Strong $(\delta, n)$-complements for semi-stable morphisms, 2018. https://arxiv.org/abs/1810.01990. $\uparrow 8,10$

[Hac14] Christopher D. Hacon, On the log canonical inversion of adjunction, Proc. Edinb. Math. Soc. (2) 57 (2014), no. 1, 139-143, DOI 10.1017/S0013091513000837. MR3165017 ^2 
[Hay99] Takayuki Hayakawa, Blowing ups of 3-dimensional terminal singularities, Publ. Res. Inst. Math. Sci. 35 (1999), no. 3, 515-570, DOI $10.2977 /$ prims/1195143612. MR1710753 $\uparrow 11$

[HK10] Christopher D. Hacon and Sándor J. Kovács, Classification of higher dimensional algebraic varieties, Oberwolfach Seminars, vol. 41, Birkhäuser Verlag, Basel, 2010. MR2675555 ^7

[HMX14] Christopher D. Hacon, James McKernan, and Chenyang Xu, ACC for log canonical thresholds, Ann. of Math. (2) 180 (2014), no. 2, 523-571, DOI 10.4007/annals.2014.180.2.3. MR3224718 个1

[HLS18] Jingjun Han, Jihao Liu, and Vyacheslav V. Shokurov, Boundedness of Singularities admitting an $\epsilon-P L T$ blow-up, 2018. Work in progress. $\uparrow 3$

[Ish00] Shihoko Ishii, The quotients of log-canonical singularities by finite groups, Singularities-Sapporo 1998, Adv. Stud. Pure Math., vol. 29, Kinokuniya, Tokyo, 2000, pp. 135-161. MR1819634 ^3

[IP01] Shihoko Ishii and Yuri Prokhorov, Hypersurface exceptional singularities, Internat. J. Math. 12 (2001), no. 6, 661687, DOI 10.1142/S0129167X0100099X. MR1875648 $\uparrow 2$

[Kol16] János Kollár, New examples of terminal and log canonical singularities, 2016. https://arxiv.org/abs/1107.2864. $\uparrow 1$

[Kol13] János Kollár, Singularities of the minimal model program, Cambridge Tracts in Mathematics, vol. 200, Cambridge University Press, Cambridge, 2013. With a collaboration of Sándor Kovács. MR3057950 ^1

[KK10] János Kollár and Sándor J. Kovács, Log canonical singularities are Du Bois, J. Amer. Math. Soc. 23 (2010), no. 3, 791-813, DOI 10.1090/S0894-0347-10-00663-6. MR2629988 个5

[KM98] János Kollár and Shigefumi Mori, Birational geometry of algebraic varieties, Cambridge Tracts in Mathematics, vol. 134, Cambridge University Press, Cambridge, 1998. With the collaboration of C. H. Clemens and A. Corti; Translated from the 1998 Japanese original. MR1658959 ^3, 4, 9

[KMM87] Yujiro Kawamata, Katsumi Matsuda, and Kenji Matsuki, Introduction to the minimal model problem, Algebraic geometry, Sendai, 1985, Adv. Stud. Pure Math., vol. 10, North-Holland, Amsterdam, 1987, pp. 283-360. MR946243 $\uparrow 11$

[KX16] János Kollár and Chenyang Xu, The dual complex of Calabi-Yau pairs, Invent. Math. 205 (2016), no. 3, 527-557, DOI 10.1007/s00222-015-0640-6. MR3539921 $\uparrow 1$

[Li17] Chi Li, K-semistability is equivariant volume minimization, Duke Math. J. 166 (2017), no. 16, 3147-3218, DOI 10.1215/00127094-2017-0026. MR3715806 ^1

[LX16] Chi Li and Chenyang Xu, Stability of Valuations and Kollr Components, 2016. https://arxiv.org/abs/1604.05398. $\uparrow 1$

[LX17] — Stability of Valuations: Higher Rational Rank, 2017. https://arxiv.org/abs/1707.05561. $\uparrow 1,4$

[MP99] D. Markushevich and Yu. G. Prokhorov, Exceptional quotient singularities, Amer. J. Math. 121 (1999), no. 6, 1179-1189. MR1719826 $\uparrow 2,5$

[PS01] Yu. G. Prokhorov and V. V. Shokurov, The first fundamental theorem on complements: from global to local, Izv. Ross. Akad. Nauk Ser. Mat. 65 (2001), no. 6, 99-128, DOI 10.1070/IM2001v065n06ABEH000366 (Russian, with Russian summary); English transl., Izv. Math. 65 (2001), no. 6, 1169-1196. MR1892905 ^1, 3

[PS09] - Towards the second main theorem on complements, J. Algebraic Geom. 18 (2009), no. 1, 151-199, DOI 10.1090/S1056-3911-08-00498-0. MR2448282 个1

[Sho93] V. V. Shokurov, A supplement to: "Three-dimensional log perestroikas" [Izv. Ross. Akad. Nauk Ser. Mat. 56 (1992), no. 1, 105-203; MR1162635 (93j:14012)], Izv. Ross. Akad. Nauk Ser. Mat. 57 (1993), no. 6, 141-175, DOI 10.1070/IM1994v043n03ABEH001579 (Russian); English transl., Russian Acad. Sci. Izv. Math. 43 (1994), no. $3,527-558$. MR1256571 $\uparrow 7,8$

[Sho96] _ 3-fold log models, J. Math. Sci. 81 (1996), no. 3, 2667-2699, DOI 10.1007/BF02362335. Algebraic geometry, 4. MR1420223 22

[Sho00] _ Complements on surfaces, J. Math. Sci. (New York) 102 (2000), no. 2, 3876-3932, DOI 10.1007/BF02984106. Algebraic geometry, 10. MR1794169 $\uparrow 2$

[Sho04] Letters of a bi-rationalist. V. Minimal log discrepancies and termination of log fips, Tr. Mat. Inst. Steklova 246 (2004), no. Algebr. Geom. Metody, Svyazi i Prilozh., 328-351 (Russian, with Russian summary); English transl., Proc. Steklov Inst. Math. 3(246) (2004), 315-336. MR2101303 $\uparrow 2$

[Xu14] Chenyang Xu, Finiteness of algebraic fundamental groups, Compos. Math. 150 (2014), no. 3, 409-414, DOI 10.1112/S0010437X13007562. MR3187625 个1, 2, 4

[Xu17] https://arxiv.org/abs/1712.01041. $\uparrow 1$

[Zar39] Oscar Zariski, The reduction of the singularities of an algebraic surface, Ann. of Math. (2) 40 (1939), 639-689, DOI 10.2307/1968949. MR0000159 $\uparrow 8$ 
Department of Mathematics, University of Utah, 155 S 1400 E, JWB 321, Salt Lake City, UT 84112, USA

E-mail address: moraga@math.utah.edu 